\title{
The Familial Dyad between Aged Patients and Filipina Caregivers in Israel: Eldercare and Bodily-based Practices in the Jewish Home
}

\author{
Keren Mazuz \\ Department of Sociology \\ Hebrew University in Jerusalem
}

\begin{abstract}
As the population in the US ages, there is increasing need to study aging In this article I describe a familial dyad between the Filipina caregiver and the Israeli aged patient. I argue that a familial dyad emerges based on bodily forms of care. This familial dyad becomes a mechanism for adaptation to and enduring of the daily and intimate encounter of a foreign caregiver and an aged dying patient. The familial dyad provides insight into the phenomenology of the care experience as a function for re-conceptualizing social relations and intra-family dynamics. This will broaden our understanding of the possible varieties of bodily-based practices and their relational repercussions as interpersonal care engagements.

The form of a familial dyad underscores the dynamism and complexity of care practices as intersubjective and corporeal modes through which one body engages the other. These care practices which are based on repetitive physical actions allow immediate first-person access to the other participants' subjective state. Thus, in an era of globalized care, the familial dyad takes form and shape at the most intimate juncture between the subjects, their corporeal and interpersonal being.
\end{abstract}

Keywords: bodily-based practices, eldercare, Filipina caregivers, empathy, family, dyad

\section{INTRODUCTION}

During the night, on September 2004, Jina called for an ambulance after Sima had trouble breathing and then accompanied her to the hospital. Sima was hospitalized and I came to visit them the next morning at 11:30 am in the ward. After an hour, the nurse entered the room and declared that visiting hours were over. While I prepared to take my things I asked Jina whether she was planning to stay again that night. She answered without hesitation "Yes, of course until Ima [mother in Hebrew] will get out" The nurse immediately added, "who else will stay here, she [Jina] is the only true family she [Sima] has".

While leaving the ward, I thought about these family-like connections and relational terms. Sima has five children and Jina isn't one of them yet the nurse considered her the "only true family" Sima has. Jina is one of the migrant workers from the Philippines and employed in Israel as caregivers for the country's aged, dying citizens who reside in their patients' homes and provide them with round-the-clock care. On what basis are these familial terms applied to them? What is their meaning and practical significance? What do they convey about the relationship between the Filipina caregiver and the Israeli patient who have different cultural modes and conceptions regarding familial behavior? It is precisely against the backdrop of such cultural and language difference that this family-like relationship emerges, unfolding through the social and bodily nature of care practices.

In this article, based on extended fieldwork among the migrant caregivers from the Philippines in Israel, I describe the family-like relations in order to broaden our understanding of the possible varieties of bodilybased practices and their relational repercussions as interpersonal care engagements. 
I argue that the family-like relationship creates a familial dyad between the Filipina caregiver and the Israeli patient based on bodily-based of care practices. This familial dyad becomes a mechanism for adaptation to and enduring of the daily and intimate encounter of a foreign caregiver and an aged dying patient. The kinship terms emerge spontaneously in the course of the interaction between caregivers and patients; their use is not the result of a decision reached in advance between them or with the patient's family members, nor as a precondition for the employment of caregivers, but as a sudden articulation that emerges through ongoing bodily closeness and intimacy. The term dyad provides insight into the phenomenology of care experience as a possible function for re-conceptualizing social relations and intra-family dynamics. As I will show below through the analysis of two ethnographies, the social and familial dynamics takes form and shape at the most intimate juncture between the subjects, and the fusion of their corporeal and emotional beings into a novel interpersonal entity which will be called here the familial dyad.

The form of a familial dyad underscores the dynamism and complexity of care practices as intersubjective and corporeal modes through which one body engages with the other. The care practices are located in the interactions between the Filipina caregiver and their patients. More precisely, in the interface between the intimacy of the aged body and the Filipina's body so the care practices are combined between the two bodies while the Filipina becomes an extension of her patient's body and daily movements. For example, they walk together at the same rhythm and the patients learn to eat according to the way the Filipina feeds them.

These care practices which are based on repetitive physical actions allow immediate first-person access to the other participants' subjective state and intention that could result in empathy. Empathy, argues Throop $(2012,408)$, is A multimodal process that not only involves perception, intellection, affect, and imagination but also the bodily, sensory, and tactile aspects of lived experience...It also highlights how particular cultural contexts, including ethnomedical and therapeutic ones, may significantly shape the expression, recognition, value, and experience of empathy as a basic existential human capacity for orienting to, interacting with, and experiencing others. In this sense, the familial dyad emerges in the most basic therapeutic context of care. The repetitive care practices assist the person being cared-for and the caregiver to orient and interact and through that to develop a sense of empathy based on recognition, gratitude, attention and physical closeness. This care practices bringing them close together as a family despite the fact that the Israeli patient and the Filipina caregiver have disparate language and cultural backgrounds and their current intimate encounters have neither a common past nor a future (in contrast, for example, to a mother-child relationship). This relationship is all the more ephemeral because it is bound in time to the remaining life span of the patient. This temporality is due to the Israel's Immigration Administration's policy which determines that after the patient dies, the caregiver is eligible for deportation to the Philippines unless she finds employment caring for another patient. Accordingly, the caregivers referred to as "daughters" by their dyad-partners are not entitled to remain in the society in which their life-work takes place nor do they have any familial rights after the patient's death. Despite these circumstances, these daily and corporeal encounters, which can extend over months or years, constitute a profoundly familial relationship, with their participants often being described as "true family". Given these circumstances, the familial dyad is not an object that is "good to think with" metaphorically but a matter of practical adjustment for both the patient and the caregiver.

Following a brief description regarding the circumstance in which the Israeli eldercare system has led to the employment of caregivers from the Philippines and an overview of the research methodology, I will describe and analyze the ethnography of care practices to get a better sense of the role of the body in such a familial dyad.

\section{The Context of Eldercare and Filipina Migrant Workers in IsRael}

The caregivers from the Philippines are mainly young, female, non-Jewish and noncitizen temporary workers employed as live-in caregivers for the country's aged, sick, and dying citizens. The option of recruiting Filipina caregivers is delivered through local placement agencies as one of the National Social Security (NSS) system services available for eldercare in Israel.

Based on the Israeli Nursing Care Law of 1988, Israeli citizens who cannot care for themselves in at least one out of five Activities of daily living (ADL) index of bodily practices - eating, dressing, walking, bathing or controlling urine or bowel movements - are considered in need of a permanent daily attendant partially paid for by state funds. This care can be obtained either in the form of a nursing institution or by home care assistance with migrant caregivers most of whom migrated from 
the Philippines (today it is also possible to recruit foreign caregivers from Nepal, India and Romania).

In contrast to Israeli caregivers who staff a nursing home, the Filipina workers provide round-the-clock care while residing in their patients' homes until the patient passes away. Within this cocoon, performing mundane and intimate life activities entails ongoing close physical interactions. Before the passing of the Law, it was accepted that the family (especially female daughters) acted as the major caretaker of elderly members. However, in the subsequent period this function has been provided by foreign guest workers.

The outflow of caregivers from the Philippines to Israel not only meets Israel's local need for eldercare, but is also part of a growing globalized economy of domestic services. Female caregivers from the Philippines are employed worldwide as caretakers for young, healthy children and families or as housecleaners (cf. in Malaysia see Chin 1998; in Kuwait see Shan et al., 2011: in Los Angeles and Rome see Parreñas 2001; in Hong Kong see Constable 1997; and in Taiwan see Cheng 2003) transforming the Philippines into the contemporary modern "empire of care" (Choy 2003).

In Israel, female migrant worker from the Philippines is known as "Filipina". A "Filipina" has become the Hebrew generic term used to describe the employment category of in-home, female non-professional caregivers who perform "bodywork" (Twigg 2000). They work according to contracts which define their temporary and low-paying conditions. The term "Filipina" in Hebrew reflects the Philippines as the caregivers' country of origin, as if the ability to provide care is part of Filipinas' natural makeup. 1 This term is based on the cultivated reputation of the Filipinas as maternal caregivers, which has actively promoted their export in the global market for domestic, geriatric and family care service.

However, in contrast to their worldwide tasks, in Israel, only working with patients known in Hebrew as se'udi is considered legal. The Hebrew term se'udi refers to a patient whose dependent condition requires the attendance of a caregiver to support in meeting the requirements of basic bodily functions. In contrast with the visually or physically challenged, who are defined according to a physical absence, the "se'udi" patient is defined as such due to the necessity of his or her dependence on the care provided by the caregiver (cf. Mazuz, 2013b). The term se'udi as used here explicitly conveys the caregivers' and the patients' physical proximity and symbiosis. This symbiosis is manifested by the close and often long-term interactions between the patient and the caregiver who, though they are two different people, engage based on bodily and tactile forms that delineate the familial dyad. Most of the Filipina work migrants I encountered during my fieldwork were not professional medical nurses. This emphasis how the globalization of domestic and care service from the Philippines illustrates how the feminization of care is based on the stereotypic notion of women as maternal, sensitive and de-professionalized caregivers. Upon arrival in Israel, they received a few hours of training at the manpower agencies before beginning their work with their patients. Occasionally, they receive further instruction from the physicians and nurses of the health maintenance organization. Most of the Filipinas' job description consists of providing basic care such as preparing meals, feeding, bathing, walking, and dressing, assisting with personal hygiene, changing diapers or taking care of toileting and cleaning the house. But over time and in tandem with the patient's deterioration they perform more responsibilities involving para-professional medical skills including dispensing medicine, installing bags to the feeding tubes or connecting the patient to a dialysis machine.

On one hand, the Filipina must develop culturally intuitive sensitivity (e.g., understanding facial and body language, sickness behaviors and gender attitudes) rapidly while caring for the patient body; a body she learns to know better than the patient's own children. As a result, she will be aware of any deteriorating change in the body which could be a matter of life and death. However, on the other hand, the Filipina's culturally intuitive abilities, which can be lifesaving, are not professionally acknowledged or compensated by the manpower agencies or the medical establishment on par with Israeli caregivers. Thus, from a bureaucratic point of view, any Filipina can be repeatedly replaced by subsequent Filipina woman.

Although the Filipina caregivers occupying the lowest ladder in the formal employment nursing structure, at the home of the patients they have become the exclusive, skillful and authorized caregivers. Over time, the Filipina foreign homecare has emerged as socially acceptable solution and respected standard of care chosen by Israeli families, despite initial fears and suspicions on the part of the patients' family members (in this aspect see Ayalon, 2009) This, notwithstanding that this involves transferring the bulk of family responsibilities to outsiders who become part of familial dyad. 


\section{Methodology}

This study is based on ethnographic research conducted between the years 2001-2008 among Filipina migrant workers in Israel. In broad terms, it examines eldercare as a local industry which includes a large number of senior citizens, governmental agencies dealing with them, and the global network of domestic Filipina caregivers who tend to the elderly. This study presents an investigation into the particular meanings of care work that expressed in practices emerging from the caregivers' daily and social lives, such as the swan folding (cf. Mazuz, 2013a) and kinship terms (cf. Mazuz, 2013b).

The research was based mostly on observation and participant-observation at the homes of thirty Israeli patients cared for by Filipina caregivers in a town located in the southern region of the country. The majority of the patients were Jewish-Israeli female citizens, widowed mothers over the age of 68 , who had migrated to Israel during the mid-1950s from North Africa. They were Hebrew and Arabic speakers, predominantly of middle to low socioeconomic status.

The caregivers had migrated largely from the rural areas in the northern islands of the Philippines. The majority of the migrants are uneducated Catholic women from a low-socioeconomic level, between the ages of 35-55, both married mothers and singles. All of them supported their families by sending material remittances back to the Philippines. The monthly salary of the Filipina caregivers is US\$500-800, paid partly by the Israeli government incentives through the agencies and partly by the families or by nursing insurance coverage. The migration process starts at the local branch of the Israeli placement agencies located in the Philippines.

The application of observation methods was particularly relevant in studying these encounters because the Filipina caregivers and the Israeli patients do not share a common language or cultural background and beliefs. In this crosscultural context, they had little verbal communication since the caregivers usually spoke no Hebrew or Arabic and the patients spoke no English or Tagalog. The Filipinas knew elementary words of conversational Hebrew and they had difficulty reading and writing in that language, particularly with respect to specialized care and treatment terminology. The patients had lack of language proficiency in English and in most of the cases Hebrew was their second language. Additionally, in cases of physical or mental impairments, the patients had difficulty communicating verbally thereby making this form of communication between the two even more difficult. As a result, speech was not central to communication between the patient and caregiver even though they lived together under the same roof. When I was present and if requested, I would translate the conversations between the two parties from Hebrew and Arabic into English and back; in some cases this function was conducted by the patients' grandchild.

Surprisingly, this difficulty that was a source of some problems also functioned as a generative source for communication of a different form, that is, the use of the most immediate alternative communicative medium: the body. The body then is the medium and locality of the care practices and ultimately of the familial dyad. Consequently, the daily tasks which were based on bodyto-body practices rendered the use of language as a tool of communication unnecessary.

Through daily care practices the Filipina migrant becomes a part, an extension, of the patient's body. Through care practices such as bathing and dressing the Filipina attends to the patient's body with her body since both constituents of the dyad learn how to use their bodies in a manner that engages and synchronizes the two bodies to perform as one and at once. So the two bodies combine in a symbiotic, mutualistic and bidirectional relationship which at times continues the movements of one's body and at other times generates them. As the following ethnographies will show, eldercare in this context is a dialogic process between two bodies and as such it is constituted in terms of social and embodied relations.

The work of caregiving was exercised through actions that were carried out repeatedly as the most useful and sensual tools of communication and comprehension. Observing the practices of caregiving convey the meaning of the familial dyad.

In the following sections, ethnographies regarding the care practices repertoire are described.

\section{CARING-For}

\section{Jina and Sima}

During three days of hospitalization, Sima was cared-for by Jina who slept next to her bed. It was not the first time that Sima was hospitalized; Jina, 36-year-old married woman and a mother of two children in the Philippines had cared for Sima since 2001 and had become experienced in what hospitalization entailed. Sima's children trusted Jina and came to visit the hospital according to their work's hours but always called Jina's phone to gain further information 
and updates about their mother's condition.

During the third day of hospitalization, I came for another visit in the morning. Sima was asleep in her bed and Jina sat next to her. Jina approached Sima and started to wash Sima's face and ears with a wet towel while Sima's eyes were still closed. Jina continued to dab Sima's lips, wiping her shoulders beneath the hospital's dress. Sima moved slowly calling "Jina, Jina". Jina bent over Sima's bed and repeated "Ima [mother], wake up, the nurse told me you can bathe today."

Jina intuitively stuck to the care habits she employed at home, positioning the pillows behind Sima's back and preparing Sima's breakfast. She fed her slowly, spoon by spoon while encouraging Sima to open her eyes by touching Sima's eyes. Feeling Jina's hand, Sima opens her eyes and began to eat the hot gruel. Sima became fully awake and Jina approached her bed, held Sima's hand saying in Hebrew "Ima Bohe [mother come]" and continued in English "come to bath, if you bath they will let us go, that's good, you want to go home, to your bed, to your neighbors". Sima smiled at Jina, and though she couldn't understand her English words she responded to Jina's request to make an effort, Sima replied, uttering slowly in Arabic "thank you, God will bless you binti [my daughter]."

Jina helped Sima out of bed and onto the plastic wheelchair, and wheeled her to the bathroom at the end of the room. The nurse came to the room and praised Jina's ability to assist Sima in such a gentle way and telling that "we prefer someone from the family to bathe the patient so she can keep her privacy and usual habits, and Jina is the right person to do so."

Jina and Sima went into the bathroom and closed the door. After completing the bath, Jina wheeled her back to the bed, lifted her into the bed while Sima banding over Jina to assist her, and Jina covered her with a blanket saying loudly "well-done, Ima [mother]" and sat next to Sima's bed and leaning against while saying loudly and happily "Now she looks good, I can see it."

It is important to note that Jina appreciated the familial terms. She told me that She felt honored when the nurse articulates her bonding with Sima as "true family," as it made her feel she belonged and in-place. Significantly, neither she nor other caregivers are forced to use familial terms. The caregiver receives emotional meaning through the familial gratification as part of the dyad's mutuality that goes beyond the financial compensation she receives for work. The dyad validates the importance of the caregiver's work which became significant in this temporal context. The familial terms were especially significant for caregivers who were first employed as servants or as aupairs in other countries. There, according to their stories, they experienced displacement with no special treatment from the host families (in this aspect see also Parreñas, 2001; Chin 1998). Thus the familial terms are unique to the working conditions in Israel as caregivers for the aged and dying citizens.

Jina attended Sima's state and needs based on her daily intelligible communication with her which was expressed in kinship terms (Ima and binti) and reinforced by the nurse description of their bonding as "true family". The care practices emerged in a dialogic process between them based on bodily-based practices: Jina's washing, dabbing, wiping, moving is followed by Sima's moving slowly and calling her; Jina then replies and continues by feeding, touching and feeling and Sima opens her eyes, eats, talks, smiles, makes an effort, and so on in a continuing process. In bidirectional relationships Jina generates movements of Sima's body who continues them over and again while paying attention to each touch, blink of the eye and facial gesture. Attention in this context creates a mutual bodily familiarity which evidences non-verbalized trust, awareness and interconnectedness: Jina knew that Sima felt better just by her attending to her appearance. "Paying attention" for Jina entails her reliance on bodily contact in attempting to understand and empathize with what Sima was experiencing. Attention, in this context, brings one into being by perceptual consciousness of the other, this is a multisensory engagement. Attending "to" and attending "with" the body are processes called "somatic modes of attention" (Csordas, 2002 p. 244). Somatic attention requires both sensory and tactile engagement as a mode of attending to each other. In this sense, we can refer to the use of familial terms as cultural elaboration of eldercare that accompany daily attention to the bodily form and movements (of the carer and cared-for).

\section{Lisa and Miriam ${ }^{2}$}

Lisa cared for Miriam from 2002 until Miriam passed away in December 2004. At first, it was like a 'blind-date' where there was nothing the two had in common, including language and cultural background. But based on day-today practices and interactions, they become familiar and relatively close, over time, despite the fact that they still did not speak the same language.

When Miriam was asked to explain her medical 
condition, she usually described it as "something sweet that eats my body". Miriam suffered from diabetes that caused loss of vision and a problem with pain in her legs. On the morning of Sunday, February 2004, I entered Miriam's house, and saw Miriam lying down in her bed and Lisa sitting next to her, holding her hands. They were still both dressed in pajamas. Miriam's three floor house was narrow, thus they were unable to get their two beds into one room. Although Lisa had her own room, she put her bed in the living-room next to Miriam's bed in order to watch and attend to Miriam's needs immediately.

Lisa whispered to me: "During the night she was in pain and the pills are not effective. I gave her a little glass of arak [Mediterranean alcoholic liquor]". Miriam opened her eyes and moaned quietly. Lisa touched Miriam's face slowly and gently, whilst holding her hand, until Miriam's eyes closed again and she fell asleep. After ten minutes, Lisa released Miriam's hand and went to the kitchen to prepare breakfast. Half an hour later, Lisa woke Miriam up, calling her "Ima [mother] wake-up you need to eat". Miriam opened her eyes, moaned, and Lisa lifted her over some pillows and began to feed her spoon after spoon. Unexpectedly, Miriam closed her eyes and bit her lips in pain. Lisa held her hand and stopped feeding her, telling her in Hebrew again "Ima [mother] I am here". Miriam's voiceless tears began to fall. She held Lisa's hands and told her in Arabic, "T'iech binti [thanks, my daughter]". Lisa relaxed Miriam by touching her face and reorganizing the pillows and helping her lie down.

When Miriam's pain overshadowed everything, she closed her eyes. Lisa responded in touching her, providing her support. Miriam's pain had become a medium for social interaction with Lisa who instinctively responded to it through the mode of touch while holding her hands. Through touch as a tactile practice they both shared the "'here and now"' of the pain experience and by doing so they both participated in temporal and spatial immediacy with the same focus of attention.

Lisa responded to Miriam's pain by employing a variety of practices which do not treat or cure but show awareness and intentionality towards the painful state. Also the use of arak exemplifies the cultural sensitivity of Lisa, since it is considered as a method of pain relief among Moroccan Jews in Israel. Although some treatments have not been medically proven as effective, their use-along with attending to the patient's bodily pain with the carer's body, sensitive attention, deliberate touching corresponding to Miriam's pain-does appear to make a difference in terms of the pain experience. The carer's touch is not taken for granted; it is part of the mutualistic relationship between them as mother and daughter. The touch, the proximity, the calling, the attention is already embedded in their bodily-based relationship.

\section{The Body of Proof}

The content of daily care between Miriam and Lisa as well as between Sima and Jina is embedded in bodily actions that are carried out repeatedly each day. As these patients' physical situation declined and their range of bodily movements narrowed, they became increasingly dependent on the Filipinas' care practices. Lisa and Jina fed, touched, held and bathed their patients. The Filipina used her hands -without gloves- as the most useful and sensual tools of apprehension and of action. There was intelligible communication between them through bodily practices. Within their hushed intervals, silences and fragments of words in three different languages, conversation and discourse were not major tools of communication.

In these encounters, given the lack of a shared verbal and cultural habitus (Bourdieu, 1977), between the Filipina caregiver and her Israeli patient, the tactile aspects of touching and bodily closeness becomes social instruments for communication that can be put to use inter-subjectively. In this context, by placing bodily-based practices at the center, the familial dyad is taking shape and form.

As both ethnographies suggest, the use of familial terms is not idiosyncratic behavior or a cross-cultural metaphor but part of their corporeal daily lived experiences. The ways in which the care practices are put together, the relative proximity between the two bodies, paying attention, the setting of parallel beds at the hospital and at home, the touching of the face, the holding of hands, the use of familiarity as noted by the nurse, are manifested through the care practices throughout they become a mother and a daughter, a single kinship unit. Thus, the physicality of the familial dyad has a communicative role that socially organizes their relationships and providing them with sense of meaning.

The familial dyad is marked, then, by the bodily practices that create relatively static, corporeal dimensions of intersubjectivity and sensual continuity. This is especially so in hand holding and touching. In the ethnographies above, the familial terms appear as the most immediate auditory utterance after or during the care practices which are based on and demand corporeal and mutual involvement. Another unique aspect of the familial terms is their inherently reciprocal, allowing the one to be a 
mother and the other to be a daughter at the same time. It is not a unidirectional relation but rather bidirectional. My objective here is not to offer an ethnomedical model of eldercare as a romanticized alternative by suggesting that the familial dyad is built as quid-pro-quo. The familial dyad never exists outside of the specific context of their encounters and it is limited to particular actors (the Filipina and patient) in a specific time and place. Nonetheless, though it is not pre-negotiated or imposed, it emerges as an integral part of the caregiver/patient relationship and serves to maintain order, respect, and status by elevating their shared experience to that of a dyad characterized by daughterhood and motherhood.

\section{CARE ANd EMPathy: BEyond wORdS}

Throop(2012) suggests through ethnography of bonesetting practice in the island of Yap the term "tactile-based empathy". The term outline that empathy is not reached only through verbal or visual modalities but as "rooted in alternate embodied and sensory forms of attuning with others" (p. 424). Throop suggest this term especially in contexts in which the capacity to gain quasi-first-person access to another's lived experience is problematic. Thus, empathy "is considered a mode of disclosing ongoing embodied forms of intersubjectivity" (p. 425).

Accordingly, the care practices that are bodily-based are crucial tools of communication especially given the disparate language and culture as a problem to gain quasifirst-person access. In this context, the care practices are not just technical or "empty" forms but materialization of "tactile-based empathy" (Throop 2012). As described above, these caregivers and care receivers used a few words in three languages - Hebrew, English, and Arabic - and still none were enough for a correct and complete conversation. At the beginning, those words were meaningless. However, they became gradually loaded with significance not through their translation but rather through the bodily care practices that accompanied them. These care practices require intuition, attention and sensitivity as essential aspects of tactile-based empathy through which the familial dyad emerges.

In their research among Indonesian nurses working in Japan, Alam \& Wulansari (2010) describe two care practices that emerge between the Indonesian nurses and the elderly Japanese patients due to the lack of language proficiency: "skinship" and empathetic caring. The term "skinship" is derived from "skin" plus the suffix of "friendship" and has surfaced as the Indonesian nurses' strategy to maintain a close relationship based on skin-to-skin practices in the absence of a shared language. "Looking at their patients as they were their own grandparents" (2010, p. 190) strengthens the Indonesians' empathetic caring.

Thus, in a theoretical manner, the application of care through physical intimacy, bodily proximity (such as skinship) and tactile moods of touching facilitated the caregiver and patient role to extend to the utmost degree of engagement, familial dyad and empathy as the main aspects of eldercare.

The familial dyad which concretely demonstrates empathy is central to our understanding of how bodily attention and practices create identifications and bonding in a reality of sickness and displacement caused by ageing and migration. The familial dyad is entangled in and acts as vectors of sociality and subjectivity; it is a new adjustable way of being-in-the-world for both the caregiver and the patient in which no one could take a part. In this context, empathy is the vehicle for adapting to the changing environment of both the cared-for and the caregiver in an era of globalized care.

\section{THE FAMILIAL DYAD CONSEQUENCES: THE FUNCTION OF THE FAMILY}

The familial dyad became a bonding essence created solely between the Filipina caregiver and the Israeli patient that no one else, including the patient's children, can take a part in. The nurse at the hospital describes Jina as the "true family" acknowledging Jina's irreplaceable position and daily stance of action which is part of eldercare.

This gives rise to the question, what are the familial dyad consequences? The familial dyad, which provides insight into the phenomenology of care experience through implementation of bodily-based practices, reconceptualizes social and intra-family dynamics of both the caregiver and the patient's family.

The patient's children do not approach the Filipina as a sister but in some cases do refer to her as a "bat Michpaha" [family member]. The Filipina caregiver frees the children from the routine and dirty, tedious and exhausting work that is considered part of eldercare. The Jewish family is reconstituted because there is one Filipina who is a noncitizen, who holds no residential or inheritance rights, and yet is essential to the operation of the Jewish family especially in the face of the modern labor system (so in Sima's case, her children could continue with their works and came to visit at the hospital). The Filipina allows the physical and social functioning of the aged parent as well as of the whole family. Consequently, the Filipina 
lubricates the gears of the Jewish family system. The Filipina enable the children to go on-consciously and practically - with their everyday lives without torments that they neglected their aged parents. Moreover, it assists them with encountering their aged parent as they would like to remember them: As one of the children told me with a somber tone "I don't want to remember my mother being diapered, she is more than that". The children's visit often occurs after their mother/father has been treated and cleaned. Thus, a Filipina foreign homecare became a standard of eldercare that Jewish-Israeli families choose and maintain as both a moral necessity and a socially acceptable option in the face of the modern labor system. The Filipina caregiver enables the children to continue functioning by transferring responsibility for ongoing bodily interactions involved in the decay of the aged body to the Filipina who, by necessity, lives and works as one unit with the patient in close physical proximity.

Another consequence affects the irreplaceable Filipina. The Filipina homecare, which shoulders the Jewish family, demands a total attendance which only a foreign female body constructed as foreign noncitizen, nonJewish temporary resident displaced in space and time, separated from her language and culture of origin, and deprived of rights and citizenship could carry repeatedly. As I concluded (Mazuz 2013, 109): "eldercare in Israel has been viewed as temporary work. Despite the fact that it shoulders and sustains the Jewish family's burden, it is based on an endless supply of a frequently changing flow of foreign women, and new, refreshed Asian bodies, until their supply becomes depleted".

Consequently, the familial dyad is located at the crossroads of global and local economics, the state regulation and bureaucratization of work migration, and cultural conceptions of eldercare. Paradoxically, this junction also fosters the most intimate and familial bond between the subjects, their corporeal and interpersonal being. These, in turn, generate an alternative conception of intra-family dynamics and burden, belonging, and empathy which are dependent on bodily-based practices and tactile interconnectedness as the core of eldercare.

\section{REFERENCES}

Alam, Bachtiar and Wulansari, Sri Ayu

2010. Creative Friction: some preliminary Considerations on the Socio-Cultural Issues Encountered by Indonesian Nurses in Japan. Bulletin of Kyushu University Asia
Center, 5, 183-192. Retrieved from: Http://hd1handle. net/2323/17937

Ayalon, L.

2009. Fears come true: the experiences of older care recipients and their family members of live-in foreign home care workers. International Psychogeriatrics, 21, 779-86

Bourdieu, Pierre

1977 Outline of a Theory of Practice. Cambridge: Cambridge University Press, 1977.

Csordas, Thomas

2002. Body/ Meaning/ Healing. New-York: Palgrave Macmillan Press.

Cheng, S.A.

2003. Rethinking the Globalization of Domestic Service: Foreign Domestics, State Control and the Politics of Identity in Taiwan. Gender \& Society 17 (2): 166-186.

Chin, Christine

1998. In Service and Servitude. New York: Columbia University Press.

Choy, C.C

2003.Empire of Care: Nursing and Migration in Filipino American History. Manila: Ateneo de Manila University Press.

Constable, Nicole

1997. Sexuality and Discipline among Filipina domestic Workers in Hong- Kong. American Ethnologist 24(3):539-558.

Parreñas, R.S.

2001. Servants of Globalization: Women, Migration and Domestic Work. California: Stanford University Press.

Shah, Nasara, Badr, Hanan, Shah, Makhdoom

2011. Foreign live-in domestic workers as caretakers of older Kuwaiti men and women: socio-demographic and health correlates, Aging \& Society, pp. 1-22.

Mazuz, Keren

2013a. Folding Paper Swans, Modeling Lives: The Rituals of Filipina Eldercare in Israel. In Medical Anthropology Quarterly 27(2): 215-232.

2013b. The State of the Family: Eldercare as a Practice of Corporal Symbiosis by Filipina Migrant Workers. In Markowitz Fran, (Ed), Ethnographic Encounters in Israel: Poetics and Ethics of Fieldwork, University of Indiana Press (pp. 97-112).

Forthcoming. Pain in-between Cultures: A Comparison between Eldercare forms in Israel. In Sternudd, Hans and Hinerman, Nate (Eds), Making sense of Pain, Inter- 
Disciplinary Press.

Throop, Jason, C.

2012. On the Varieties of Empathic Experience: Tactility, Mental Opacity, and Pain in Yap, Medical Anthropology Quarterly, 26(3): 408-430.

Twigg, Julia

2000. Carework as a Form of Bodywork, Ageing and Society 20 (4):389-411. 